\section{Circadian hepatocyte clocks keep synchrony in the absence of a master pacemaker in the suprachiasmatic nucleus or other extrahepatic clocks}

\author{
Flore Sinturel, ${ }^{1,2,3,4,12}$ Pascal Gos, ${ }^{5,12}$ \\ Volodymyr Petrenko, 1,2,3,4 Claudia Hagedorn, ${ }^{6}$ \\ Florian Kreppel, ${ }^{6}$ Kai-Florian Storch, 7,10 \\ Darko Knutti, ${ }^{7,11}$ Andre Liani, ${ }^{5}$ Charles Weitz, ${ }^{7}$ \\ Yann Emmenegger, ${ }^{8}$ Paul Franken, ${ }^{8}$ \\ Luigi Bonacina, ${ }^{9}$ Charna Dibner, ${ }^{1,2,3,4}$ \\ and Ueli Schibler 5
}

\begin{abstract}
${ }^{1}$ Department of Medicine, Division of Endocrinology, Diabetes, Nutrition, and Patient Education, University Hospital of Geneva, 1211 Geneva, Switzerland; ${ }^{2}$ Department of Cell Physiology and Metabolism, Faculty of Medicine, University of Geneva, 1211 Geneva, Switzerland; ${ }^{3}$ Institute of Genetics and Genomics of Geneva (iGE3), 1211 Geneva, Switzerland; ${ }^{4}$ Diabetes Center, Faculty of Medicine, University of Geneva, 1211 Geneva, Switzerland; ${ }^{5}$ Department of Molecular Biology, Sciences III, University of Geneva, 1211 Geneva, Switzerland; ${ }^{6}$ Center of Biomedical Education and Research, University Witten/ Herdecke, 58448 Witten, Germany; ${ }^{7}$ Department of Neurobiology, Harvard Medical School, Boston, Massachusetts 02115, USA; ${ }^{8}$ Center for Integrative Genomics, University of Lausanne, 1015 Lausanne, Switzerland; ${ }^{9}$ Department of Applied Physics, University of Geneva, 1211 Geneva, Switzerland
\end{abstract}

It has been assumed that the suprachiasmatic nucleus (SCN) synchronizes peripheral circadian oscillators. However, this has never been convincingly shown, since biochemical time series experiments are not feasible in behaviorally arrhythmic animals. By using long-term bioluminescence recording in freely moving mice, we show that the SCN is indeed required for maintaining synchrony between organs. Surprisingly, however, circadian oscillations persist in the livers of mice devoid of an SCN or oscillators in cells other than hepatocytes. Hence, similar to SCN neurons, hepatocytes can maintain phase coherence in the absence of Zeitgeber signals produced by other organs or environmental cycles.

Supplemental material is available for this article.

Received November 25, 2020; revised version accepted January 14, 2021.

[Keywords: circadian gene expression; in vivo bioluminescence recording; liver; suprachiasmatic nucleus]

Present addresses: ${ }^{10}$ Douglas Mental Health University Institute, Department of Psychiatry, McGill University, Montreal, Quebec, Canada; ${ }_{11}^{11}$ Bayer Consumer Care AG, Basel, Switzerland.

${ }^{12}$ These authors contributed equally to this work.

Corresponding authors: ueli.schibler@unige.ch, charna.dibner@unige.ch Article published online ahead of print. Article and publication date are online at http://www.genesdev.org/cgi/doi/10.1101/gad.346460.120. Freely available online through the Genes \& Development Open Access option.
The mammalian circadian timing system is composed of a master pacemaker in the brain's suprachiasmatic nucleus (SCN) and cell-autonomous clocks in most tissues (Nagoshi et al. 2004; Welsh et al. 2004). These oscillators consist of two interlocked transcriptional-translational feedback loops (TTFLs) (Schibler et al. 2015; Cox and Takahashi 2019). In the primary TTFL, Period and Cryptochrome genes (Per1, Per2, Cry1, and Cry2) are activated by CLOCK-BMAL1 heterodimers. PER and CRY proteins then assemble into large repressor complexes that inhibit the CLOCK-BMAL1 activity (Schmalen et al. 2014; Aryal et al. 2017). Consequently, the levels of PER and CRY transcripts and proteins decrease, and a new round of PER and CRY expression can ensue. In a secondary TTFL REV-ERB $\alpha, \beta$ repressors counteract the stimulation of Bmal1 and Clock transcription by ROR $\alpha, \beta, \gamma$ activators.

Since circadian clocks can measure a $24-h$ day only approximately, they must be synchronized daily. The SCN is synchronized primarily by light-dark cycles (Patton and Hastings 2018; Hastings et al. 2019). The strong phase coupling among neurons and between neurons and astrocytes establish circadian phase coherence even in cultured SCN explants (Yamazaki et al. 2000; Liu et al. 2007; Herzog et al. 2017). Peripheral clocks are synchronized by feeding-fasting rhythms (Damiola et al. 2000; Stokkan et al. 2001; Crosby et al. 2019), blood-borne signaling molecules, and body temperature cycles (for review, see Schibler et al. 2015), but they rapidly loose phase coherence in vitro (Welsh et al. 2004). Using a bioluminescence imaging method, Tahara et al. (2012) found that about half of SCN-lesioned animals still displayed rhythms in liver, kidney, and submaxillary gland. However, their method involved anesthesia and is thus not appropriate for continuous recording over multiple days. Here, we adapted the RT-Biolumicorder technique (Saini et al. 2013) to the monitoring of circadian gene expression in freely moving mice for many weeks to examine the impact of the SCN and feeding-fasting cycles on peripheral clocks. Moreover, we monitored circadian gene expression in behaviorally arrhythmic mice harboring clocks exclusively in hepatocytes.

\section{Results and Discussion}

To extend the recording time of the original RT-Biolumicorder technology (Saini et al. 2013), we provided luciferin in the drinking water instead of through an Alzet micro-osmotic pump (Fig. 1A-C; Supplemental Fig. S1A-C). The two delivery methods were then compared for the monitoring of whole-body Per2::luciferase expression in hairless mice entrained by a skeleton photoperiod. As shown in Figure 1, A-C, the bioluminescence displayed high-amplitude circadian oscillations with both methods (Fig. 1A). Spontaneous locomotor and drinking activities showed similar temporal profiles in the presence or absence of the pump (Fig. 1B,C; Supplemental Fig. S1D). Likewise, we observed robust circadian bioluminescence cycles with both delivery methods when recording a mouse whose liver was transduced by a Rev-erba-luciferase adenoviral vector

(C) 2021 Sinturel et al. This article, published in Genes \& Development, is available under a Creative Commons License (Attribution-NonCommercial 4.0 International), as described at http://creativecommons.org/licenses/by-nc/4.0/. 

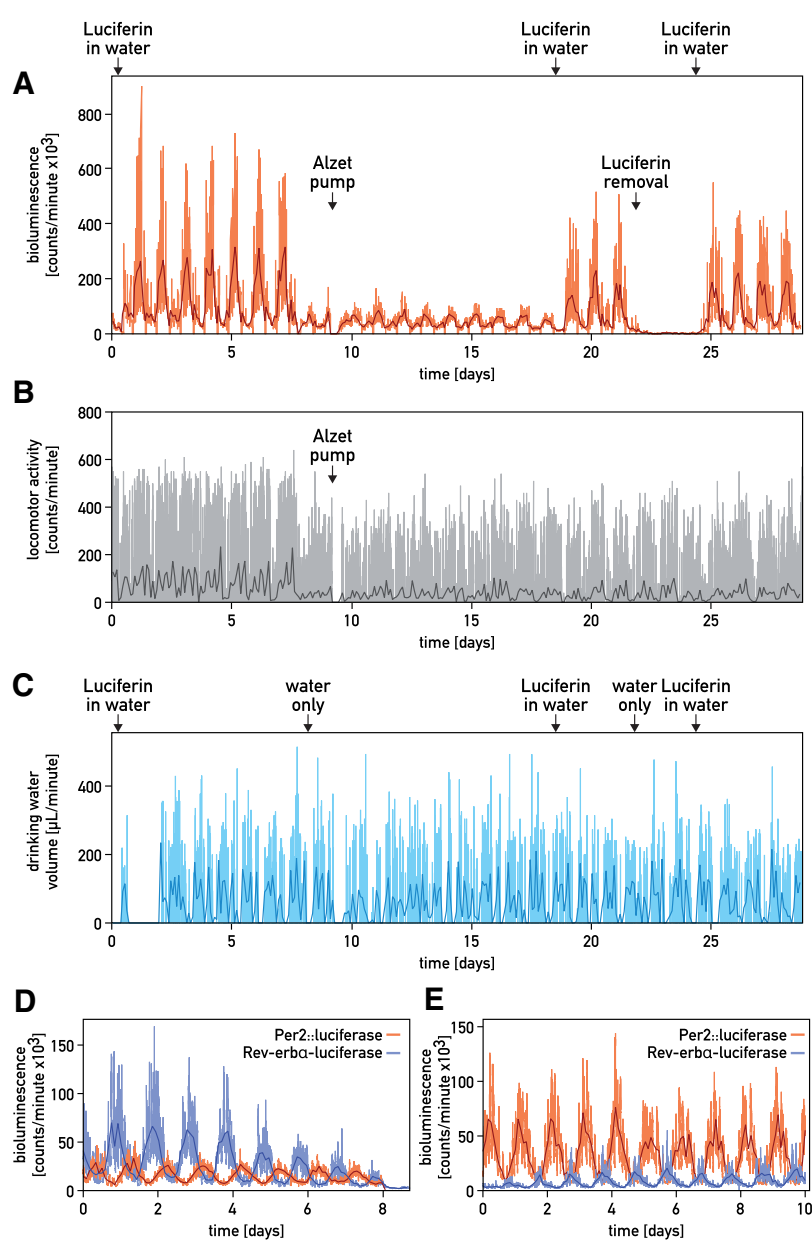

E
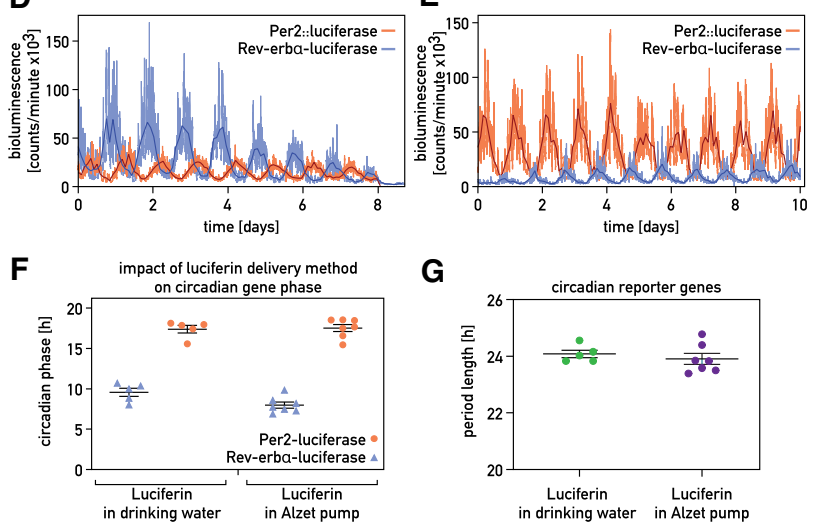

G

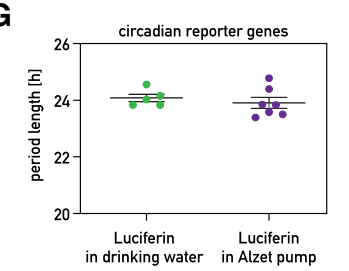

Figure 1. Long-term recording of circadian whole-body bioluminescence. $(A-C)$ Whole-body recording of a hairless Per2::luc mouse entrained by a skeleton photoperiod. Luciferin was delivered as indicated. $(A)$ Bioluminescence. $(B)$ Spontaneous locomotor activity. $(C)$ Drinking activity. (Orange, gray, and light blue lines in $A-C$ ) Raw data (1-min photon counts, 1-min activity counts, and 1-min water consumption, respectively), (darker lines in $A-C$ ) 120-min time-binned traces. $(D, E)$ Whole-body Per2::luciferase and hepatic Rev-erba-luciferase recordings from two different animals kept in constant darkness and receiving luciferin through Alzet pumps $(D)$ or with the drinking water (E). (Light lines) Raw data, (darker lines) 120-min time-binned traces. $(F, G)$ Relationship between the phases $(F)$ and the period lengths $(G)$ of Per2::luciferase and hepatic Reverba-luciferase expression of mice receiving luciferin in the drinking water $(n=5)$ or by an Alzet micro-osmotic pumps $(n=7)$. Mice were kept in constant darkness.

(Supplemental Fig. S1A-C). Importantly, animals maintained in constant darkness and receiving luciferin in the drinking water displayed a nearly antiphasic expression of Rev-erba-luciferase and Per2::luciferase (Fig. 1D,E) with indistinguishable period length or phase (Fig. 1F,G). Therefore, the drinking rhythms, generated by rest-activity cycles, did not interfere dramatically with the bioluminescence recordings. Fast Fourier Transform (FFT) analysis showed that the amplitude of the FFT peak at $24 \mathrm{~h}$ was similar when luciferin was delivered with the drinking water (Supplemental Fig. S1E), and the luciferin delivery method did not significantly affect the accuracy of period length (Supplemental Fig. S1F).

We next performed experiments with PER2::luciferase reporter mice (Yoo et al. 2004) whose SCN was intact or ablated by stereotaxic lesions (Fig. 2A,B; Supplemental Fig. S2A-F). As expected, locomotor activity was lost in SCN-lesioned but not in sham-operated animals kept in constant darkness (D/D) (Fig. 2C; Supplemental Fig. S3). To examine the impact of feeding-fasting rhythms on locomotor activity and bioluminescence, PER2::luc mice were maintained in $\mathrm{D} / \mathrm{D}$ and fed according to the regimens indicated on top of Figure 2A. While the locomotor activity of SCN-lesioned mice was arrhythmic in animals fed ad libitum, it showed clear rhythmicity in animals subjected to $12 \mathrm{~h} / 12 \mathrm{~h}$ feeding-fasting rhythms (Fig. 2D-G). As shown in Figure 2A and Supplemental Figure $\mathrm{S} 2 \mathrm{C}, \mathrm{D}$, whole-body bioluminescence (i.e., the sum of photons emitted by many organs) showed high-amplitude oscillations in sham-operated mice. In contrast, these rhythms were strongly dampened in SCN-lesioned mice, suggesting that the SCN is required for the synchronization of oscillators among different tissues. Imposing feeding rhythms synchronized PER2::luc expression in both sham-operated and SCN-lesioned mice (Fig. 2A,B,E; Supplemental Fig. S2C-F). In agreement with previous studies (Damiola et al. 2000; Le Minh et al. 2001; Saini et al. 2013), the phase of circadian gene expression was shifted more rapidly in SCN-lesioned as compared with sham-operated mice (Supplemental Fig. S2G). The period lengths and amplitudes of the FFT peaks at $24 \mathrm{~h}$ were similar in SCN-proficient and -deficient mice when animals were subjected to feeding-fasting cycles (Fig. 2F-I). Unexpectedly, the overall bioluminescence was markedly higher in recordings of SCN-lesioned as compared with sham-operated mice (Supplemental Fig. S2H). Therefore, signals depending directly or indirectly on the SCN appeared to reduce, rather than increase, the magnitude of PER2::luciferase expression in peripheral tissues.

Since a residual rhythmicity of PER2::luc expression could still be discerned in SCN-lesioned mice, we suspected that some cellular oscillators could still be in phase in these animals. To examine this conjecture for liver, we transduced liver cells of sham-operated and SCN-lesioned mice with the adenoviral Rev-erba-luciferase vectors via tail vein injection (Fig. 3A,B; Supplemental Fig. S4A-E; Saini et al. 2013). Hepatic Rev-erba-luciferase expression oscillated robustly in both SCN-proficient and -deficient mice monitored in constant condition, irrespective of whether luciferin was administered through the drinking water (Fig. 3B) or via an Alzet pump (Supplemental Fig. S4B-E). A similar observation was made for hepatic Bmall-luciferase expression (Supplemental Fig. S4E-G). The FFT analysis of the bioluminescence profiles (Fig. $3 \mathrm{C}, \mathrm{D})$ revealed no statistically significant differences for the FFT peak at $24 \mathrm{~h}$ in SCN-proficient and -deficient animals (Fig. 3E). As expected, the SCN-lesioned mice displayed significantly lower amplitudes of the FFT peak at $24 \mathrm{~h}$ for the locomotor activity (Fig. 3F). Although hepatic Rev-erba-luciferase expression in SCN-lesioned mice showed a somewhat larger variability of the period length and a slightly increased width of the 24-h oscillations (Fig. 
A

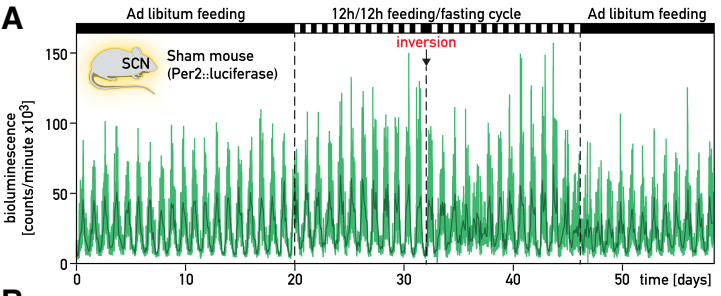

B
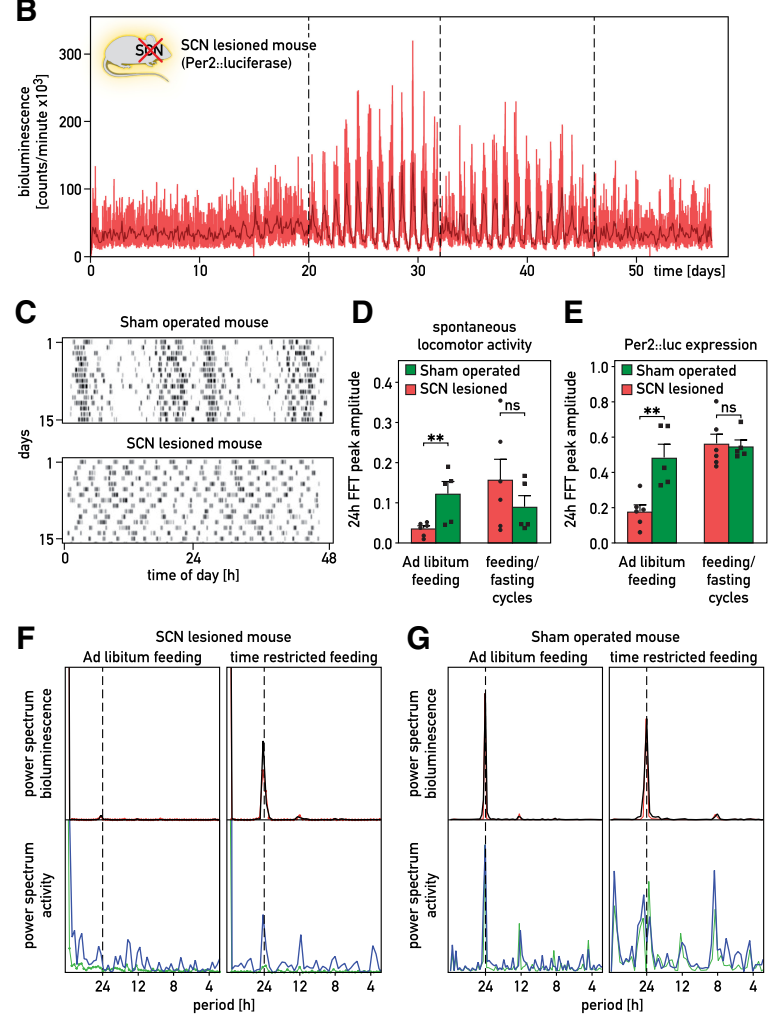

G

Sham operated mouse

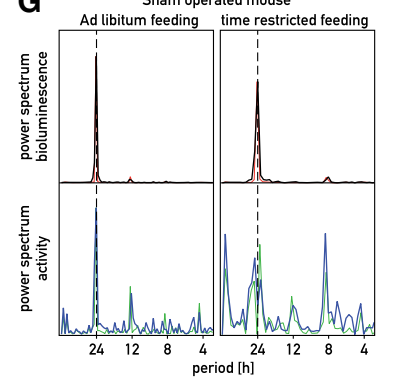

I

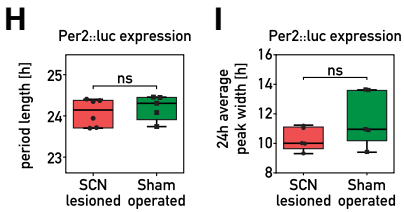

Figure 2. The SCN is required for the synchronization of peripheral clocks among organs. $(A, B)$ Whole-body bioluminescence for shamoperated $(A)$ and SCN-lesioned $(B)$ Per2::luc mice kept in constant darkness. The feeding regimens are reported above the graphs. The spontaneous locomotor activities of the animals are shown in Supplemental Figure S2A,B. (Thin lines) Raw data (1 min photon counts), (bold lines) 120-min time-binned traces. (C) Actograms of spontaneous locomotor activity of the sham-operated mouse used in $A$ and the SCN-lesioned mouse used in $B$. After surgery, mice were housed in 12-h/12-h light/dark (LD) cycles and then recorded during $15 \mathrm{~d}$ in constant darkness (D/D). $(D, E)$ Amplitude of the FFT peak in the circadian range $(\approx 24 \mathrm{~h})$ of locomotor activities $(D)$ and of Per2::luciferase expression $(E)$ of $S C N$-lesioned and sham-operated mice subjected to different feeding regimens (means \pm SEM, $n \geq 5$ per group). $(F, G)$ Periodograms (FFT analysis) of bioluminescence and locomotor activity recorded for the sham-operated mouse displayed in $A$ and the SCNlesioned mouse displayed in $B$. (Red and green lines) Raw data (1min counts), (black and blue lines) integrated data over 120 points. $(H, I)$ Period length from FFT analysis $(H)$ and average oscillation width $(I)$ in sham-operated and SCN-lesioned animals subjected to feeding-fasting cycles. Whiskers of the box plots range from the minimum to the maximum values. The median values are indicated by the horizontal segment within the box, $n \geq 5$ per group. Statistical tests for $D, E, H$, and $I$ were two-tailed Mann-Whitney test. $\left.{ }^{* *}\right) P<$ 0.01 , (ns) nonsignificant.
A

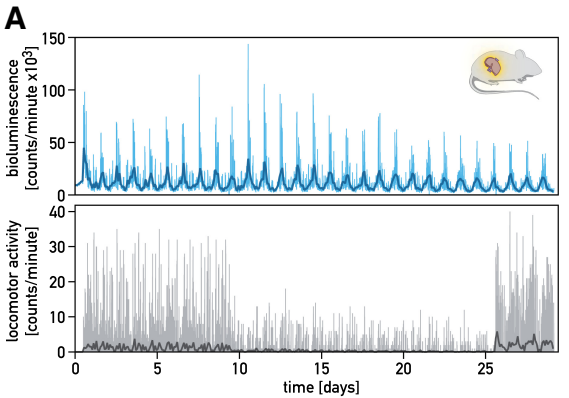

B

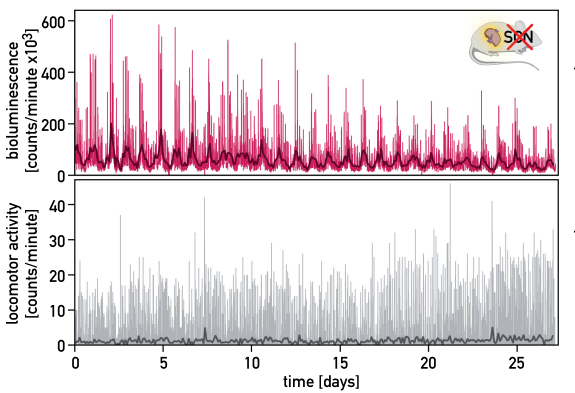

D
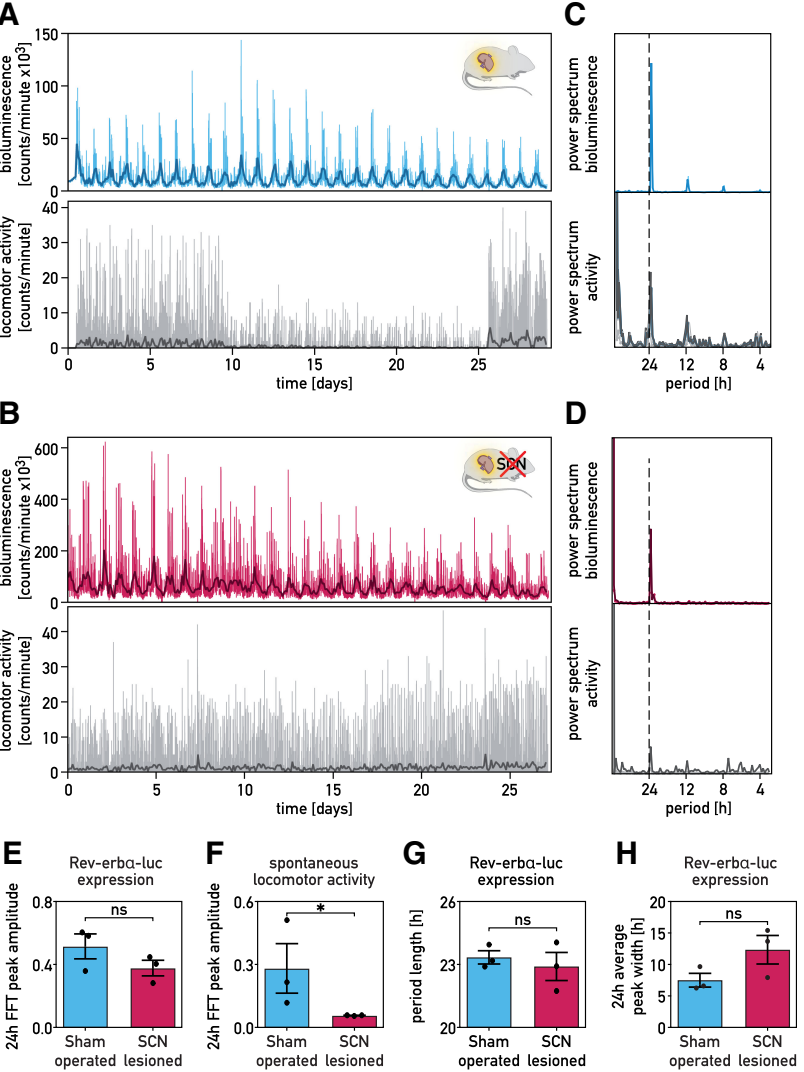

Figure 3. Hepatocyte oscillators maintain synchrony in SCN-lesioned animals. $(A, B)$ Recordings of circadian Rev-erba-luciferase expression in the livers of sham-operated (blue; $A$ ) and SCN-lesioned $($ pink $B)$ mice receiving luciferin in the drinking water. Mice transduced with the Adv-Rev-erba-luc vector were shaved in a dorsal region over the liver. Forty-eight hours after transduction, bioluminescence was monitored in constant darkness (food and water ad libitum). Spontaneous locomotor activity profiles monitored simultaneously are shown below the bioluminescence tracings. The drop of the signal for locomotor activity in the middle of the recording was caused by the obstruction of the IR detector by litter piled up by the mouse. (Thin colored and gray lines ) Raw data (1-min counts), (bold lines) 120-min time-binned traces. (C,D) Periodograms (FFT analysis) of the data shown in $A$ and $B$. (Thin colored and gray lines) Raw data (1-min counts), (bold lines) 120-min time-binned traces. $(E, F)$ Amplitudes of the FFT peaks in the circadian range of hepatic Rev-erba-luciferase expression $(E)$ and spontaneous locomotor activity rhythms $(F)$ of SCN-lesioned and sham-operated mice maintained in constant conditions (means \pm SEM, $n=3$ per group). The statistical test was a one-tailed Mann-Whitney test. $\left(^{*}\right) P<0.05$, (ns) nonsignificant. $(G, H)$ Period lengths of Rev-erba-luciferase expression $(G)$ and average oscillation width in the circadian range $(\approx 24 \mathrm{~h})(H)$ of shamoperated and SCN-lesioned animals maintained in constant conditions (means \pm SEM, $n=3$ per group). The statistical test was a twotailed Mann-Whitney test. (ns) Nonsignificant.

3G,H), these differences were not statistically significant. As the amplitude and peak width were not significantly different between sham-operated and SCN-lesioned mice fed ad libitum or subjected to a feeding-fasting regimen (Fig. 3E,H), the SCN did not markedly enhance the amplitude of hepatocyte oscillators and/or their intercellular coupling within the liver. Given that the efficiency of adenoviral transductions varied somewhat between animals, we could not directly compare the magnitude of bioluminescence generated by the livers of sham-operated and SCN-lesioned mice. 
To examine whether liver cells remain synchronized in the absence of nonhepatic signals, we studied mice (kept in constant darkness) harboring clocks exclusively in hepatocytes. These animals, dubbed "hepatocyte clock-only mice," contain a Bmal1-allele carrying a lox-stop signal be-

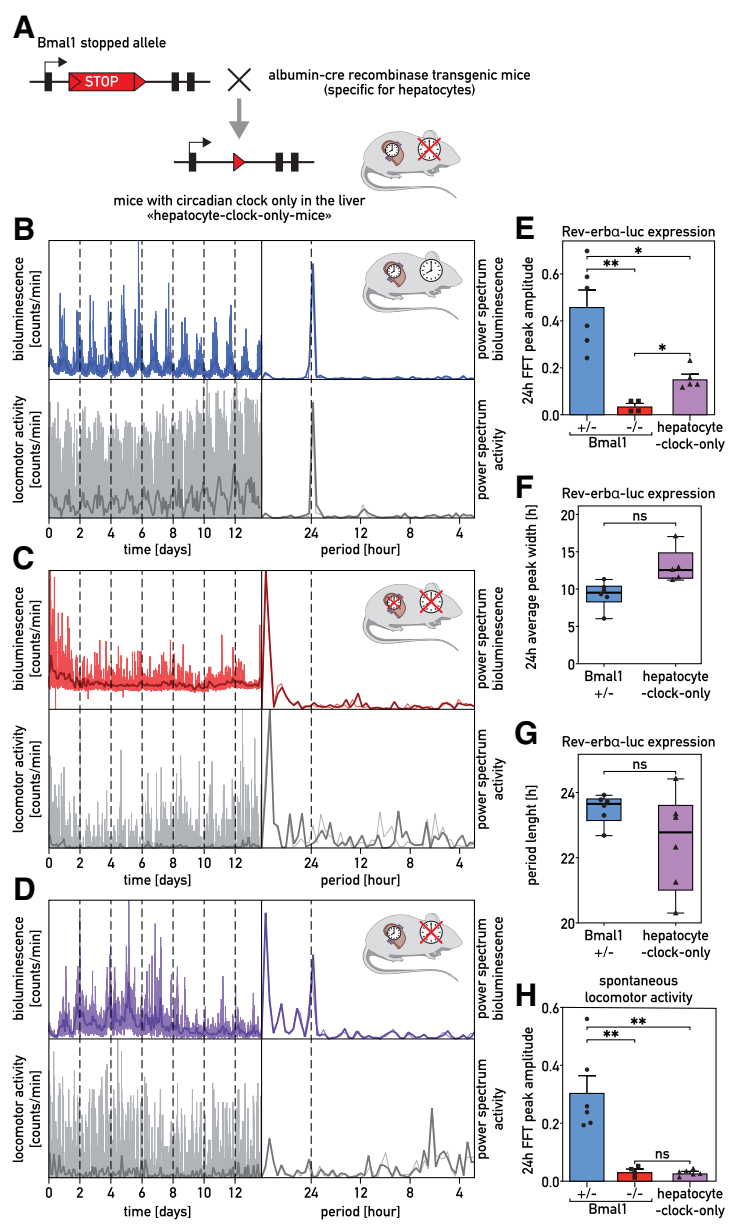

Figure 4. The livers of "hepatocyte clock-only mice" keep partial phase coherence. $(A)$ Scheme of the lox-stop allele used to generate "hepatocyte clock-only mice." $(B-D)$ Recordings of hepatic expression of Rev-erba-luciferase in Bmal1 ${ }^{+/}$mice (blue; $B$ ), Bmal1 ${ }^{-/-}$ mice (red; $C$ ), and "hepatocyte clock-only mice" (violet; $D$ ) receiving luciferin in the drinking water. Mice were transduced with the AdvRev-erba-luc expression vector, and bioluminescence was recorded under constant conditions (D/D, food and water ad libitum). Spontaneous locomotor activity profiles are shown below the bioluminescence tracings. The FFT analysis of the bioluminescence and locomotor activity recordings are represented at the right of the bioluminescence and activity tracings. (Thin colored and gray lines) Raw data (1-min counts), (bold lines) 120-min time-binned traces. (E) Amplitude of the FFT peaks in the circadian range, determined for hepatic Reverba-luciferase expression in $\mathrm{Bmal1}^{+{ }^{+-}}$mice, $\mathrm{Bmal1}^{-/-}$mice, and "hepatocyte clock-only mice." $(F)$ Width of the oscillations for hepatic Rev-erba-luciferase expression in the circadian range $(\approx 24 \mathrm{~h})$ in $B$ mal1 ${ }^{+/-}$and "hepatocyte clock-only mice." $(G)$ Period length of $B$ mal1 ${ }^{+/-}$mice and "hepatocyte clock-only mice." $(H)$ Amplitude of the FFT peaks in the circadian range, determined for the spontaneous locomotor activity rhythms of $B$ mal1 ${ }^{+/-}$mice, $B$ mal1 $^{-1-}$ mice, and "hepatocyte clock-only mice." The statistical test for $E$ and $H$ was a Kruskal-Wallis test corrected by FDR (means \pm SEM, $n \geq 4$ per group), and for $F$ and $G$ a two-tailed Mann-Whitney test $\left(n \geq 4\right.$ per group). $\left({ }^{* *}\right) P$ $<0.01,\left({ }^{*}\right) P<0.05$, (ns) nonsignificant. $(F, G)$ Whiskers of the box plots represent minimum to maximum values with median value indicated by the horizontal segment within the box. tween exon 5 and 6 that can be deleted with a Cre recombinase specifically expressed in hepatocytes (Fig. 4A; Supplemental Fig. S5A,B). In parallel, we used $\mathrm{Bmal1}^{+}-$mice, in which Bmal1 is expressed throughout the body, and arrhythmic Bmal1-/- mice. As shown in Figure 4B,C and Supplemental Figure S5C-K, bioluminescence recordings of $\mathrm{Bmal1}^{+/-}$and $\mathrm{Bmal1}^{-/-}$mice revealed circadian and arrhythmic Rev-erba-luciferase expression, respectively, in the livers of these animals. Strikingly, we observed rhythmic oscillations of hepatic Rev-erba-luciferase expression in "hepatocyte clock-only mice," but, as expected, not in Bmal1 ${ }^{-1-}$ mice devoid of a Cre recombinase (Fig. 4D). "Hepatocyte clock-only mice" showed less stable rhythms of Rev-erba-luciferase expression than $\mathrm{Bmal1}^{+/-}$ or SCN-lesioned animals. However, these oscillations were statistically highly significant (Fig. 4E-G) and revealed a dominant peak at around $24 \mathrm{~h}$ in all periodograms (Supplemental Fig. S5L-P). As anticipated, neither Bmal1 ${ }^{-/}$mice nor "hepatocyte clock-only mice" showed behavioral rhythms (Fig. 4H). Hence, hepatocytes were partially synchronized in behaviorally arrhythmic mice that harbor circadian oscillators exclusively in hepatocytes.

In contrast to previous reports (Guo et al. 2006; Koronowski et al. 2019), our findings show that the SCN is not required for maintaining phase coherence between hepatocytes. Based on photon imaging of anesthetized, SCN-lesioned PER2::luc mice (Tahara et al. 2012) and the bioluminescence recording of tissue explants from SCN-lesioned PER2::luc mice (Yoo et al. 2004), similar conclusions were reached.

In "hepatocyte clock-only mice," the synchronization of liver clocks was less complete than in SCN-lesioned mice. Conceivably, in SCN-lesioned mice, signals emitted by liver cells other than hepatocytes (or nonhepatic tissues) might have participated in the synchronization of hepatocyte clocks. Indeed, a recent study provided direct evidence for intercellular communication by different cell types in controlling rhythmic liver gene expression (Guan et al. 2020). In contrast to the results reported here, Koronowski et al. (2019) did not observe rhythmic gene expression in "hepatocyte clock-only mice" kept in constant darkness when comparing liver mRNA levels between individual animals. Presumably, the phases of hepatic circadian gene expression were different between these individuals. The data presented here thus exemplify the importance of real-time recording when studying cyclic gene expression in behaviorally arrhythmic animals.

In conclusion, and as illustrated in Figure 5, our study revealed that the SCN master pacemaker is required for keeping phase coherence between different organs in mice kept under constant conditions. However, within the liver, hepatocytes remain perfectly synchronized in the absence of an SCN and maintain some phase coherence even in mice in which circadian oscillators function exclusively in hepatocytes. The identification of signaling pathways involved in the phase coupling between hepatocyte oscillators will be an enticing future endeavor.

\section{Materials and methods}

Animal care

Male hairless mice (strain code 477; formerly SKH1 mice with strain code 313) were purchased from Charles River, and PER2::Luc mice were generously provided by Joseph S. Takahashi (University of Texas Medical Center, Dallas, TX) (Yoo et al. 2004). Hairless PER2::Luc knock-in mice were 


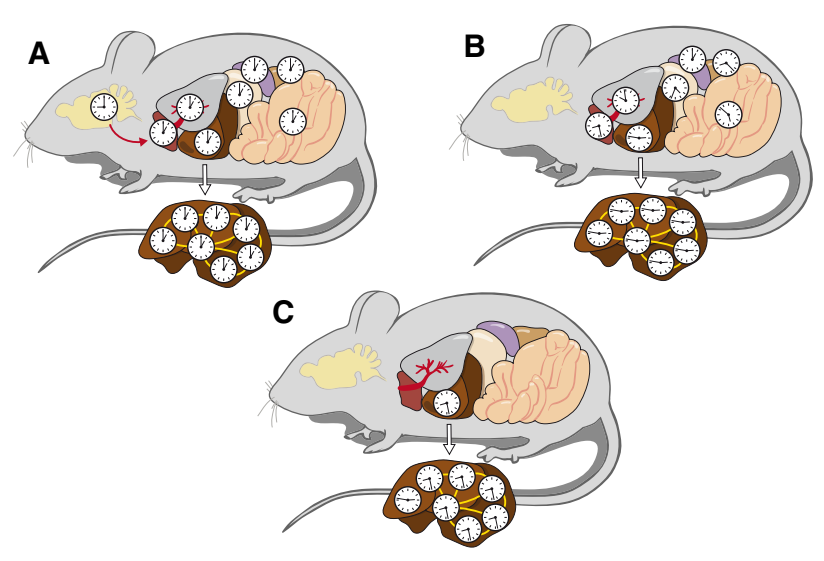

Figure 5. Scheme of circadian hepatocyte clock synchrony in control animals and in absence of the SCN or other extrahepatic clocks. (A) In intact, behaviorally rhythmic mice, the central pacemaker in the SCN synchronizes circadian oscillators to similar phases in peripheral organs (red arrow). The phases of peripheral clocks are somewhat delayed with regard to the phase of the SCN (Damiola et al. 2000). (B) In SCN-lesioned, behaviorally arrhythmic mice, the circadian clocks of different peripheral organs have different phases, but within the liver the cellular oscillators remain synchronized. $(C)$ In behaviorally arrhythmic "hepatocyte clock-only mice," a large proportion of hepatocyte clocks are still in phase. The findings illustrated in $B$ and $C$ suggest that circadian hepatocyte oscillators are phase coupled, as indicated by the yellow lines connecting hepatocyte clocks.

generated by crossing SKH1 mice with PER2::Luc mice. C57BL/6J mice homozygous for the conditional Bmal1 stopped allele (Bmal1 "stopped" mice) (Petrenko et al. 2020) were bred with mice carrying a Cre recombinase transgene under the control of the albumin promoter [albumin-Cre, B6.Cg-Tg(Alb-cre)21Mgn/J] (Postic et al. 1999), generating "hepatocyte clock-only mice" with Bmal1 expression restricted to the hepatocytes (for details, see the Supplemental Material). Bmal1-/- ("stopped") and $\mathrm{Bmal1}^{+} /^{-}$heterozygote littermates were used as controls in the experiments.

All animals were housed according to Swiss standard conditions. All the experiments were performed on healthy mice, with normal immune status, and approved by the Veterinary Offices of the Cantons of Geneva and Vaud. When indicated, feeding or light-dark cycles were imposed during bioluminescence recording. Entrainment with skeleton photoperiods (Fig. 1; Supplemental Fig. S1) was performed as described previously with two light pulses of $30 \mathrm{~min}$ at $12 \mathrm{~h}$ intervals (Friedrich and Soriano 1991; Oishi et al. 2002).

\section{SCN ablation}

Bilateral lesion of the SCN was performed stereotaxically (Kopf Instruments 963LS) under ketamine/xylazine anesthesia (intraperitoneal injection, 75 and $10 \mathrm{mg} / \mathrm{kg}$, at a volume of $8 \mathrm{~mL} / \mathrm{kg}$ ) as described (Saini et al. 2013). The success of the experiments was controlled by recording spontaneous locomotor activity in individual cages equipped with infrared sensors (Visonic) in free-running conditions (constant darkness, food and water provided ad libitum) for at least $10 \mathrm{~d}$.

\section{Generation of circadian reporter luciferase-expressing adenoviral expression vectors}

Rev-erba-luciferase and Bmal1-luciferase-expressing adenoviral constructs have been previously described (Kreppel et al. 2002; Saini et al. 2013).

\section{Luciferin delivery and tail vein injection}

For the experiments with micro-osmotic pumps, pumps (model 2001, blue flow moderator 0002489, Alzet, Charles River) were filled with 90 $\mathrm{mg} / \mathrm{mL}$ luciferin (306A NF-FF free-acid firefly D-Luciferin, Nanolight
Technology, Prolume Ltd.) and implanted into the intraperitoneal cavity under anesthesia, as described previously (Saini et al. 2013). For longterm recordings, luciferin (XenoLight D-Luciferin, potassium salt; PerkinElmer, $4.9 \mathrm{mM}$ stock concentration in sterile double-distilled water) was diluted in tap water at a final concentration of $1.56 \mathrm{mg} / \mathrm{mL}$ and provided in the drinking water. Delivery of adenoviral-luciferase reporters $\left(2.5 \times 10^{10}\right.$ to $1 \times 10^{11}$ adenoviral particles $)$ into the liver was performed as described previously (Saini et al. 2013). After 40-48 h of recovery, the animals were transferred to the RT-Biolumicorder, and the bioluminescence monitoring started.

\section{RT-Biolumicorder measurements and data analysis}

The RT-Biolumicorder (Lesa-Technology SA) has been designed and engineered as described in Saini et al. (2013), and details are in the Supplemental Material.

Original data of bioluminescence, activity, and drinking temporal profiles were collected at 1-min intervals (raw data are in Supplemental Table S1) and analyzed using the software Igor Pro v. 8.0.4 (WaveMetrics, Inc.). A custom function was applied to bin the data points summing their values over 120-min intervals. This binning time procedure was especially required for analyzing the activity traces, which might contain multiple voids. Igor Pro built-in function DSP Periodogram was applied on each binned trace to compute the power spectrum

$$
P=\frac{\mid\left. a b s[F(\text { signal })]\right|^{2}}{N},
$$

where $F$ indicates the Fast Fourier Transform operator, and $N$ is the total number of samples. The data sets were automatically detrended prior to undergoing FFT. The power spectra were successively normalized by the sum of their values. An automatic procedure was applied to fit with a Gaussian curve the power spectrum in an interval centered around the 24-h period. The fitted values for the Gaussian peak amplitude and central position were used for the statistical comparisons among different data sets. Note that the fitted values were retained for the analysis only if the circadian peak at $24 \mathrm{~h}$ resulted statistically significant. To determine the width of 24-h oscillations, we applied a Gaussian multipeak fit procedure to the complete time traces and successively calculated the average peak FWHM and standard deviation for each condition. For the calculation of phase angle changes as a function of time after inversion of the feeding regimen, the peaks in the oscillatory bioluminescence response of a given animal were fitted with a Gaussian function to automatically retrieve the positions of their maxima. The time shift with respect to an ideal 24-h daily cycle was computed by subtracting to the $n$th peak position, the position of the first peak, and the total number of daily cycles. $(n-1) \times 24 \mathrm{~h}$. The phase shift was finally calculated by setting $24 \mathrm{~h}=360^{\circ}$.

\section{Statistical analyses}

For comparison across different experimental groups, statistical significance was assessed with statistical tests in Prism 8 (GraphPad). The statistical test used, the significance values, and the number of experimental replicates for each experimental group are reported in the figure legends. Grouped data were plotted in Prism 8 as mean \pm SEM.

\section{RNA analysis by quantitative real-time RT-PCR}

Whole-cell RNAs from liver, kidney, spleen, and brown adipose tissue (BAT) were purified as previously described (Sinturel et al. 2017; details in the Supplemental Material).

For the quantification of specific transcripts, cDNAs were synthesized from total RNAs using random hexamers and SuperScript II reverse transcriptase (Thermo Fisher Scientific) following the supplier's instructions. The cDNAs were quantified by real-time PCR-amplification in a LightCycler 480 (Roche), using the SYBR Green Master Mix (Roche). Mean levels were calculated from triplicate PCR assays for each sample and normalized to the amounts of Gapdh transcripts (Supplemental Table S2). 


\section{Competing interest statement}

A.L. is a stockholder of Lesa-Technology SA, which makes the RT-Biolumicorder commercially available.

\section{Acknowledgments}

We thank M. Stoffel, ETH Zürich, for providing Albumin Cre mice; J. Takahashi, University of Texas Southwestern Medical Center, Dallas, for providing PER2::luc mice; and N. Roggli for the artwork. Work in the laboratory of U.S. was supported by the Swiss National Science Foundation (SNSF; grants 31-113565 and 31-128656/1), the European Research Council (ERC-2009-AdG-TIMESIGNAL-250117), the State of Geneva, and the Louis Jeantet Foundation of Medicine. F.S. was supported by a Swiss Society of Endocrinology and Diabetology (SGED) Young Investigator grant. Work in the laboratory of C.D. was supported by SNSF grants 31003A_166700/1 and 310030_184708/1, the Vontobel Foundation, the Novartis Consumer Health Foundation, the Swiss Life Foundation, the European Foundation for the Study of Diabetes/Novo Nordisk Program for Diabetes Research in Europe, and the Olga Mayenfisch Foundation. Work in the laboratory of C.W. was supported by a grant from the National Institutes of Health (NS055831) and a fellowship to D.K. from the SNSF. Work in the laboratory of P.F. was supported by SNSF grant 31003A_146694 and the State of Vaud. Work in the laboratory of F.K. and C.H. was supported by the Center of Biomedical Research and Education (ZBAF) at Witten/Herdecke University. L.B.'s work was supported by the State of Geneva.

Author contributions: F.S., C.D., P.F., and U.S. designed the experiments. F.S., P.G., and V.P. did the mouse husbandry and performed the RT-Biolumicorder recording and biochemical experiments. F.K. and C.H. produced the adenoviral vectors. A.L. engineered and constructed the RT-Biolumicorder. K.F.S., D.K., and C.W. produced the Bmal1-lox-stop mice. Y.E. and P.F. performed the SCN lesions. L.B. processed the RT-Biolumicorder data. F.S., C.D., and U.S. wrote the first draft of the manuscript that was edited and approved by all coauthors.

\section{References}

Aryal RP, Kwak PB, Tamayo AG, Gebert M, Chiu PL, Walz T, Weitz CJ. 2017. Macromolecular assemblies of the mammalian circadian clock. Mol Cell 67: 770-782.e6. doi:10.1016/j.molcel.2017.07.017

Cox KH, Takahashi JS. 2019. Circadian clock genes and the transcriptional architecture of the clock mechanism. J Mol Endocrinol 63: R93-R102. doi:10.1530/JME-19-0153

Crosby P, Hamnett R, Putker M, Hoyle NP, Reed M, Karam CJ, Maywood ES, Stangherlin A, Chesham JE, Hayter EA, et al. 2019. Insulin/IGF-1 drives PERIOD synthesis to entrain circadian rhythms with feeding time. Cell 177: 896-909.e20. doi:10.1016/j.cell.2019.02.017

Damiola F, Le Minh N, Preitner N, Kornmann B, Fleury-Olela F, Schibler U. 2000. Restricted feeding uncouples circadian oscillators in peripheral tissues from the central pacemaker in the suprachiasmatic nucleus. Genes Dev 14: 2950-2961. doi:10.1101/gad.183500

Friedrich G, Soriano P. 1991. Promoter traps in embryonic stem cells: a genetic screen to identify and mutate developmental genes in mice. Genes Dev 5: 1513-1523. doi:10.1101/gad.5.9.1513

Guan D, Xiong Y, Trinh TM, Xiao Y, Hu W, Jiang C, Dierickx P, Jang C, Rabinowitz JD, Lazar MA. 2020. The hepatocyte clock and feeding control chronophysiology of multiple liver cell types. Science 369: 1388-1394. doi:10.1126/science.aba8984

Guo H, Brewer JM, Lehman MN, Bittman EL. 2006. Suprachiasmatic regulation of circadian rhythms of gene expression in hamster peripheral organs: effects of transplanting the pacemaker. J Neurosci 26: 64066412. doi:10.1523/JNEUROSCI.4676-05.2006

Hastings MH, Maywood ES, Brancaccio M. 2019. The mammalian circadian timing system and the suprachiasmatic nucleus as its pacemaker. Biology (Basel) 8: 13. doi:10.3390/biology 8010013

Herzog ED, Hermanstyne T, Smyllie NJ, Hastings MH. 2017. Regulating the suprachiasmatic nucleus ( $\mathrm{SCN}$ ) circadian clockwork: interplay between cell-autonomous and circuit-level mechanisms. Cold Spring Harb Perspect Biol 9: a027706. doi:10.1101/cshperspect.a027706

Koronowski KB, Kinouchi K, Welz PS, Smith JG, Zinna VM, Shi J, Samad M, Chen S, Magnan CN, Kinchen JM, et al. 2019. Defining the inde- pendence of the liver circadian clock. Cell 177: 1448-1462.e14. doi:10.1016/j.cell.2019.04.025

Kreppel F, Biermann V, Kochanek S, Schiedner G. 2002. A DNA-based method to assay total and infectious particle contents and helper virus contamination in high-capacity adenoviral vector preparations. Hum Gene Ther 13: 1151-1156. doi:10.1089/104303402320138934

Le Minh N, Damiola F, Tronche F, Schütz G, Schibler U. 2001. Glucocorticoid hormones inhibit food-induced phase-shifting of peripheral circadian oscillators. Embo j 20: 7128-7136. doi:10.1093/emboj/20.24 .7128

Liu AC, Welsh DK, Ko CH, Tran HG, Zhang EE, Priest AA, Buhr ED, Singer $\mathrm{O}$, Meeker K, Verma IM, et al. 2007. Intercellular coupling confers robustness against mutations in the SCN circadian clock network. Cell 129: 605-616. doi:10.1016/j.cell.2007.02.047

Nagoshi E, Saini C, Bauer C, Laroche T, Naef F, Schibler U. 2004. Circadian gene expression in individual fibroblasts: cell-autonomous and selfsustained oscillators pass time to daughter cells. Cell 119: 693-705. doi:10.1016/j.cell.2004.11.015

Oishi K, Fukui H, Sakamoto K, Miyazaki K, Kobayashi H, Ishida N. 2002. Differential expressions of mPer1 and mPer2 mRNAs under a skeleton photoperiod and a complete light-dark cycle. Brain Res Mol Brain Res 109: 11-17. doi:10.1016/S0169-328X|02)00457-6

Patton AP, Hastings MH. 2018. The suprachiasmatic nucleus. Curr Biol 28: R816-R822. doi:10.1016/j.cub.2018.06.052

Petrenko V, Stolovich-Rain M, Vandereycken B, Giovannoni L, Storch KF, Dor Y, Chera S, Dibner C. 2020. The core clock transcription factor BMAL1 drives circadian $\beta$-cell proliferation during compensatory regeneration of the endocrine pancreas. Genes Dev 34: 1650-1665. doi:10.1101/gad.343137.120

Postic C, Shiota M, Niswender KD, Jetton TL, Chen Y, Moates JM, Shelton KD, Lindner J, Cherrington AD, Magnuson MA. 1999. Dual roles for glucokinase in glucose homeostasis as determined by liver and pancreatic $\beta$ cell-specific gene knock-outs using Cre recombinase. I Biol Chem 274: 305-315. doi:10.1074/jbc.274.1.305

Saini C, Liani A, Curie T, Gos P, Kreppel F, Emmenegger Y, Bonacina L, Wolf JP, Poget YA, Franken P, et al. 2013. Real-time recording of circadian liver gene expression in freely moving mice reveals the phase-setting behavior of hepatocyte clocks. Genes Dev 27: 1526-1536. doi:10 $.1101 /$ gad.221374.113

Schibler U, Gotic I, Saini C, Gos P, Curie T, Emmenegger Y, Sinturel F, Gosselin P, Gerber A, Fleury-Olela F, et al. 2015. Clock-Talk: interactions between central and peripheral circadian oscillators in mammals. Cold Spring Harb Symp Quant Biol 80: 223-232. doi:10.1101/ sqb.2015.80.027490

Schmalen I, Reischl S, Wallach T, Klemz R, Grudziecki A, Prabu JR, Benda C, Kramer A, Wolf E. 2014. Interaction of circadian clock proteins CRY1 and PER2 is modulated by zinc binding and disulfide bond formation. Cell 157: 1203-1215. doi:10.1016/j.cell.2014.03 .057

Sinturel F, Gerber A, Mauvoisin D, Wang J, Gatfield D, Stubblefield JJ, Green CB, Gachon F, Schibler U. 2017. Diurnal oscillations in liver mass and cell size accompany ribosome assembly cycles. Cell 169: 651-663.e14. doi:10.1016/j.cell.2017.04.015

Stokkan KA, Yamazaki S, Tei H, Sakaki Y, Menaker M. 2001. Entrainment of the circadian clock in the liver by feeding. Science 291: 490-493. doi:10.1126/science.291.5503.490

Tahara Y, Kuroda H, Saito K, Nakajima Y, Kubo Y, Ohnishi N, Seo Y, Otsuka M, Fuse Y, Ohura Y, et al. 2012. In vivo monitoring of peripheral circadian clocks in the mouse. Curr Biol 22: 1029-1034. doi:10 .1016/j.cub.2012.04.009

Welsh DK, Yoo SH, Liu AC, Takahashi JS, Kay SA. 2004. Bioluminescence imaging of individual fibroblasts reveals persistent, independently phased circadian rhythms of clock gene expression. Curr Biol 14: 2289-2295. doi:10.1016/j.cub.2004.11.057

Yamazaki S, Numano R, Abe M, Hida A, Takahashi R, Ueda M, Block GD, Sakaki Y, Menaker M, Tei H. 2000. Resetting central and peripheral circadian oscillators in transgenic rats. Science 288: 682-685. doi:10 $.1126 /$ science.288.5466.682

Yoo SH, Yamazaki S, Lowrey PL, Shimomura K, Ko CH, Buhr ED, Siepka SM, Hong HK, Oh WJ, Yoo OJ, et al. 2004. PERIOD2::LUCIFERASE real-time reporting of circadian dynamics reveals persistent circadian oscillations in mouse peripheral tissues. Proc Natl Acad Sci 101: 5339-5346. doi:10.1073/pnas.0308709101 


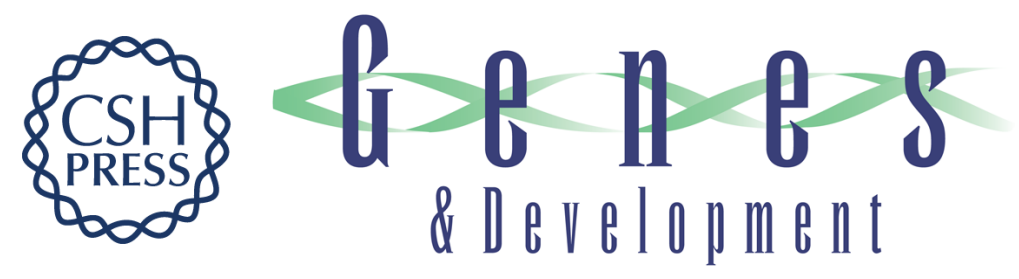

\section{Circadian hepatocyte clocks keep synchrony in the absence of a master pacemaker in the suprachiasmatic nucleus or other extrahepatic clocks}

Flore Sinturel, Pascal Gos, Volodymyr Petrenko, et al.

Genes Dev. 2021, 35: originally published online February 18, 2021

Access the most recent version at doi:10.1101/gad.346460.120

\section{Supplemental http://genesdev.cshlp.org/content/suppl/2021/02/17/gad.346460.120.DC1 \\ Material}

Related Content Peripheral clocks tick independently of their master

Anna-Marie Finger and Achim Kramer

Genes Dev. March , 2021 35: 304-306

References This article cites 27 articles, 13 of which can be accessed free at:

http://genesdev.cshlp.org/content/35/5-6/329.full.html\#ref-list-1

Articles cited in:

http://genesdev.cshlp.org/content/35/5-6/329.full.html\#related-urls

Creative This article, published in Genes \& Development, is available under a Creative Commons

Commons

License (Attribution-NonCommercial 4.0 International), as described at

License http://creativecommons.org/licenses/by-nc/4.0/.

Email Alerting

Receive free email alerts when new articles cite this article - sign up in the box at the top

Service right corner of the article or click here.

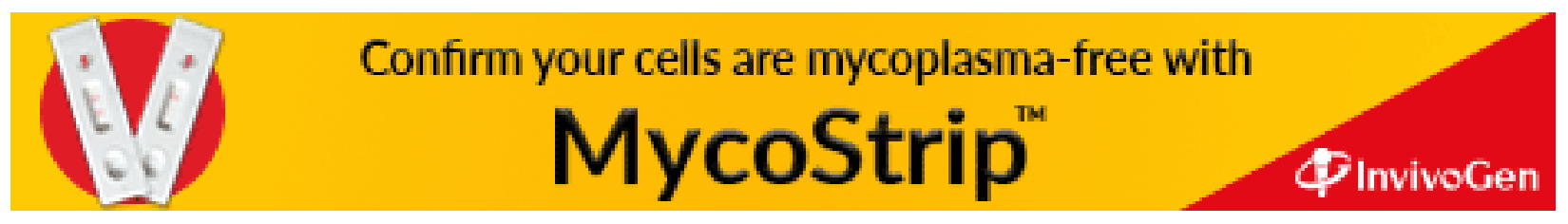

